\section{Buchrezension zu: The Autotrophic Biorefinery}

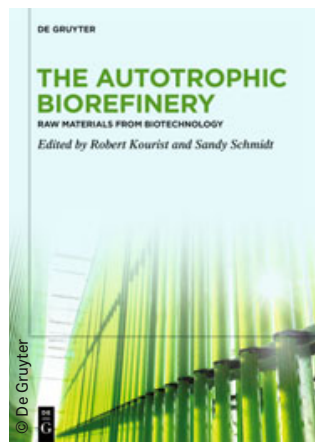

The Autotrophic Biorefinery

Raw Materials from

\section{Biotechnology}

Robert Kourist und Sandy

Schmidt (Hrsg.)

404 S., 98 Abb., 20 Tab., De Gruyter, 2021. $H C, 95,59 €$

ISBN: 978-3-11-054988-1

Auch als E-Book erhältlich

DOI: $10.1007 / \mathrm{s} 12268-022-1698-5$

(C) Der Autor 2022

Nach Johann Wolfgang von Goethe ist „die Natur allein unendlich reich, und sie allein bildet den großen Künstler“. Wir wissen, der Reichtum ist in ihrer Diversität begründet und das beruht auf einem kontinuierlichen Prozess der Evolution und Anpassung an die Umwelt. So sind mitunter effiziente, aber vor allem flexible Systeme entstanden. Die Natur und deren Prozesse, Eigenschaften und Aktivitäten sich zum Vorbild zu nehmen, nachzuahmen oder zu übertragen auf aktuelle (bio)technologische Fragestellungen ist in den letzten Jahrzenten immer weiter vorangeschritten. Hier kann man gut den Begriff der Bioraffinerie einordnen, in dem es darum geht, nachhaltig Biomasse zu verwerten und in diverse, uns nützliche, wertvolle Produkt(ström)e zu wandeln. Dabei spielen vor allem die verschiedenen Organismen und deren metabolische Fähigkeiten eine zentrale Rolle.

In dem Werk werden vor allem autotrophe Mikroorganismen und deren Fähigkeiten im Sinne einer nachhaltigen Nutzung von Kohlendioxid beschrieben. Die $\mathrm{CO}_{2}{ }^{-}$
Fixierung in Kombination mit mannigfaltigen Energiequellen eröffnet spannende biotechnologische Chancen, welche verständlich in 14 Kapiteln vermittelt werden. Diese befassen sich mit Methoden, welche auch auf andere Fragestellungen übertragbar sind, biotechnologischen Prozessen, Anwendungen und SubstratProdukt-Beziehungen sowie ökonomischen Betrachtungen. Es werden aktuelle Beispiele der (industriellen) Forschung auf hohem Niveau, aber zugleich sehr anschaulich nähergebracht. Hier sind zahlreiche, farbige Abbildungen aber auch die eingefügten Boxen mit wichtigen Definitionen hervorzuheben. Die einzelnen Kapitel können unabhängig voneinander genutzt und erarbeitet werden.

Zusammenfassend kann ich sagen, dass das in Englisch verfasste Buch vor allem für die Ausbildung von Masterstudierenden geeignet und sicher auch ein guter Start für Doktorand:innen ist, um sich in das Thema einzulesen. Der modulare Charakter und die allgemeine Schreibweise erlaubt es, das Buch in Abschnitten zu nutzen und neben den autotrophen Prozessen auch für weitere Lebensweisen und Aufgaben heranzuziehen. Es ist preislich angemessen und für eine breite Gruppe von Studierenden im Bereich der Biologie, (Bio-)Chemie, Biotechnologie und verwandten Disziplinen nutzbar.

Dirk Tischler, Ruhr-Universität Bochum, dirk.tischler@rub.de

Diese Rezension erscheint Open Access. *
* Funding note: Open Access funding enabled and organized by Projekt DEAL. Open Access: Dieser Artikel wird unter der Creative Commons Namensnennung 4.0 International Lizenz veröffentlicht, welche die Nutzung, Vervielfältigung, Bearbeitung, Verbreitung und Wiedergabe in jeglichem Medium und Format erlaubt, sofern Sie den/die ursprünglichen Autor(en) und die Quelle ordnungsgemäß nennen, einen Link zur Creative Commons Lizenz beifügen und angeben, ob Änderungen vorgenommen wurden. Die in diesem Artikel enthaltenen Bilder und sonstiges Drittmaterial unterliegen ebenfalls der genannten Creative Commons Lizenz, sofern sich aus der Abbildungslegende nichts anderes ergibt. Sofern das betreffende Material nicht unter der genannten Creative Commons Lizenz steht und die betreffende Handlung nicht nach gesetzlichen Vorschriften erlaubt ist, ist für die oben aufgeführten Weiterverwendungen des Materials die Einwilligung des jeweiligen Rechteinhabers einzuholen. Weitere Details zur Lizenz entnehmen Sie bitte der Lizenzinformation auf

http://creativecommons.org/licenses/ by $/ 4.0 /$ deed.de. 